\title{
USING WAVELET TRANSFORM TO EXTRACT FREQUENCY CONTENT IN ELECTRIC FIELD RADIATED BY UNUSUAL LIGHTNING ACTIVITY
}

\author{
Suraj Neupane*, Shriram Sharma*, and Puja Sharma** \\ *Department of Physics, Amrit Campus, Tribhuvan University, Kathmandu, Nepal. \\ ${ }^{* *}$ Department of Physics, Birendra Multiple Campus, Tribhuvan University, Chitwan, Nepal.
}

\begin{abstract}
In this frequency spectrum electric fields radiated by the unusual lightning activities have been computed using the wavelet transform technique. The unusual lightning activities have very recently been identified activities and are very poorly understood among the lightning community. As the electric fields are very recently identified and are measured in time domain, to the best of our knowledge, their frequency content has not been studied as of today. To understand the physics of the discharge mechanism of such events, the frequency domain information plays a significant role. In order to extract frequency domain information from the time domain electric field signatures the wavelet transform technique has been employed. For the purpose, the electric field pertinent to the unusual activity, has been divided into two parts namely main activity and the preceding opposite polarity field change. From the computation, it is found that the opposite-polarity field change radiates energy in the spectral range of $2 \mathrm{kHz}$ to $173 \mathrm{kHz}$ whereas, the main activity predominantly radiates in the frequency range $2 \mathrm{kHz}$ to $162 \mathrm{kHz}$. Such a wider spectral range that the unusual activities radiate have not been reported for the other known activities such as positive and negative return strokes. Evidently, the unusual events have some unique origin of discharge unlike the known activities. Further, as the unusual events were noticed in the temperate region (Uppsala, Sweden) and Sub-tropical climatic zone (Kathmandu, Nepal), it should have some common source of origin between two regions.
\end{abstract}

Keywords: Unusual lightning; Electromagnetic field; Frequency; Wavelet transform.

\section{INTRODUCTION}

Electromagnetic fields radiated by lightning flashes were causing deleterious impact on the living being on the ground from centuries. People were found to be using different preventive measures in order to mitigate such lifethreatening hazard, however only from a few centuries ago researchers were successful in understanding this phenomenon. With the development of science and technology, many inventions were made mainly in the area of wireless technology, space and aviation industry. Later, lightning flashes were found to be major threat to those electronic instruments and wireless technology. Scientist had identified that the radiated frequency of lightning signatures to be influential factor to affect such instruments ${ }^{1}$.

Lightning activities have, in general, been divided into two categories. The lightning activities that transport electrical charge between the cloud and ground are termed as lightning ground flashes, whereas, the lightning activities that transport the electrical charges between two regions of cloud are termed as cloud flashes. The ground flashes are further divided into the positive type and the negative type whereas, the cloud flashes are also divided into three type namely inter cloud, intra-cloud and cloud to air discharges. The electric fields pertinent to the known lightning

Author for correspondence: Suraj Neupane, Department of Physics, Amrit Campus, Tribhuvan University, Kathmandu, Nepal.

E-mail: physicist.surajneupane@gmail.com

Received: 7 Nov 2020; First Review: 19 dec 2020; Second Review: 24 dec 2020; Accepted: 26 Dec 2020

Doi: https://doi.org/10.3126/sw.v14i14.34988 
activities have extensively been studied and can be found in the literature. However, the newly discovered unusual activities are not very well understood as the information about these activities is not available in the literature. The unusual activities have a uniqueness in a sense that the electrical field radiated by them is different from those radiated by the know ground flashes and cloud flashes, though they appear to be similar to those of positive ground flashes. The uniqueness of the electric fields pertinent to the unusual activities can found in the earlier work ${ }^{2}$. Unlike the positive return stroke, the unusual activities produce and an electric field comprising of a main event preceded by the opposite polarity leader type pulse. A pronounced oppositepolarity pulse just prior to the main event of lightning signals is termed as Unusual lightning. The duration of opposite polarity pulses are some tens of microseconds and extend toward the positive $y$-axis. The main events are relatively slower negative (according to the atmospheric electricity sign convention) electromagnetic discharge, which appear on the negative y-axis on electric field plots of lightning. For the first time, such lightning signals were observed by the second author of this paper during a measurement campaign conducted in Uppsala, Sweden in 2009, similar lightning signals were observed in 2015 during a measurement campaign conducted in Kathmandu, Nepal; which was mentioned in the paper ${ }^{2}$. Authors have also mentioned that such lightning events were not included in any literature before, so it is new for the researcher working on lightning field.

Many researches, for instance; $1,3,4,5$, were carried out in order to calculate the frequency radiated by different types of lightning signals. Techniques such as narrow band receivers (NBRs) and wide band waveforms digitizers (WWDs) were employed by the researchers to calculate the frequency content of lightning signals, however due to different demerits in low and high frequency calculation, those techniques were not fully acknowledged to be appropriate $^{3}$. The development of Fourier transform tool in algorithm form led scientist and researcher to use this technique. However, after the Fourier transform of such transient irregular lightning signal, time information is lost, so it is impossible to predict when and where such frequency occurred. After the development of wavelet transform in algorithm form, researchers started calculating frequency using this technique. Wavelet technique has been found to be one of the most sophisticated tools to calculate the frequency content on any irregular signals ${ }^{4}$. The mathematical expression to calculate discrete wavelet transform is shown in equation (1).

$w_{t}(s, \tau)=\sum_{t=0}^{N-1} f(t) \psi_{s, \tau}^{*}(t) \delta t$

Where $*$ denotes complex conjugation. This equation shows how a function $f(t)$ is decomposed into a set of basis functions $\psi_{s, \tau}(t)$ called the wavelets. Shifting and scaling is done on each segment of the data for the calculation of range of frequency radiated by the signals, which is given as equation (2).

$\psi_{s, \tau}(t)=\frac{1}{\sqrt{s}} \psi\left(\frac{t-\tau}{s}\right)$

Where $s$ and $\tau$ are scaling and translating parameter ${ }^{6}$. Among the infinite possible mother wavelet for analysis of irregular signals, Derivative of Gaussian (DOG) was identified in the research paper ${ }^{4}$, as the most stable wavelet so far for the calculation of frequency content of lightning signals. Since it captures both positive and negative oscillations of the signal as a separate peak in wavelet power. The equation which represents the DOG mother wavelet is shown in equation (3).

$\psi_{0}(t)=\frac{(-1)^{m+1}}{\sqrt{\Gamma\left(m+\left(\frac{1}{2}\right)\right)}} \frac{d^{m}}{d t^{m}}\left(e^{-\frac{t^{2}}{2}}\right)$

The value of $\mathrm{m}$ is 2 for the derivative of the Gaussian wavelet. Wavelet transform techniques calculate the frequency at the different scale at the same point of a signal, so the value of frequency is not of exact value but a range of values ${ }^{4}$.

Wavelet transform technique have been used extensively in last two decade by many researchers, such as; ${ }^{3,7,8,9}$, for lightning electromagnetic signals. However, only in 2011 it was comprehensively used in the paper ${ }^{4}$ to calculate the frequency content of different types of lightning electromagnetic signals or different events associated within a single event. This research paper can be considered as a landmark work in the field of frequency calculation of 
lightning signals using wavelet transform algorithms. Similarly, in 2014 wavelet technique was used in the research work ${ }^{5}$ for the frequency calculation of first peak associated with different types of lightning signals.

In this research work we calculate the frequency content on unusual lightning activities. Since, this type of lightning is new for the scientific community working in the field of lightning and it is important to know the frequency it radiates.

\section{INSTRUMENTATION AND METHODOLOGY}

The lightning electric field signatures were obtained using a parallel plate antenna, which was installed $12 \mathrm{~m}$ above the surface of ground on roof top of a building at Kathmandu, Nepal. The instrumental setup used in our research to record lightning electromagnetic signals was designed and made at Uppsala University, Sweden. The measuring set up consists of three components: 1) parallel plate antenna, 2) buffer circuit and 3) digitizer along with storage. The capacitance of parallel plate antenna was set to $60 \mathrm{pF}$. The output of the buffer amplifier was fed to the digital storage oscilloscope by using properly terminated RG-58 coaxial cable. The RC decay time constant of the field measuring system was 13 $\mathrm{ms}$, which is large enough to record lightning electric field waveforms and are influenced by the instrumental decay. The data were digitized in a 4-channel Picoscope being operated in pre-trigger mode and saved in a pc.

In order to carry out this research, 16 unusual electric field temporal signals from four different days (4 signals from each day) were chosen. Those all signals were recorded in the summer thunderstorms in 2015 on April 15, 16 \& 17 and on June 10 from the measuring station situated at Kathmandu, Nepal. The sampling rate and time interval of the plot is not kept uniform during the measurement for all those signals. The time duration of $200 \mathrm{~ms}$ was set to capture the lightning signals, which is large enough to cover all events associated with each signal. DOG was selected as a mother wavelet for the analysis in our research, as this wavelet is the most stable one so far for the analysis of lightning signal ${ }^{4}$.

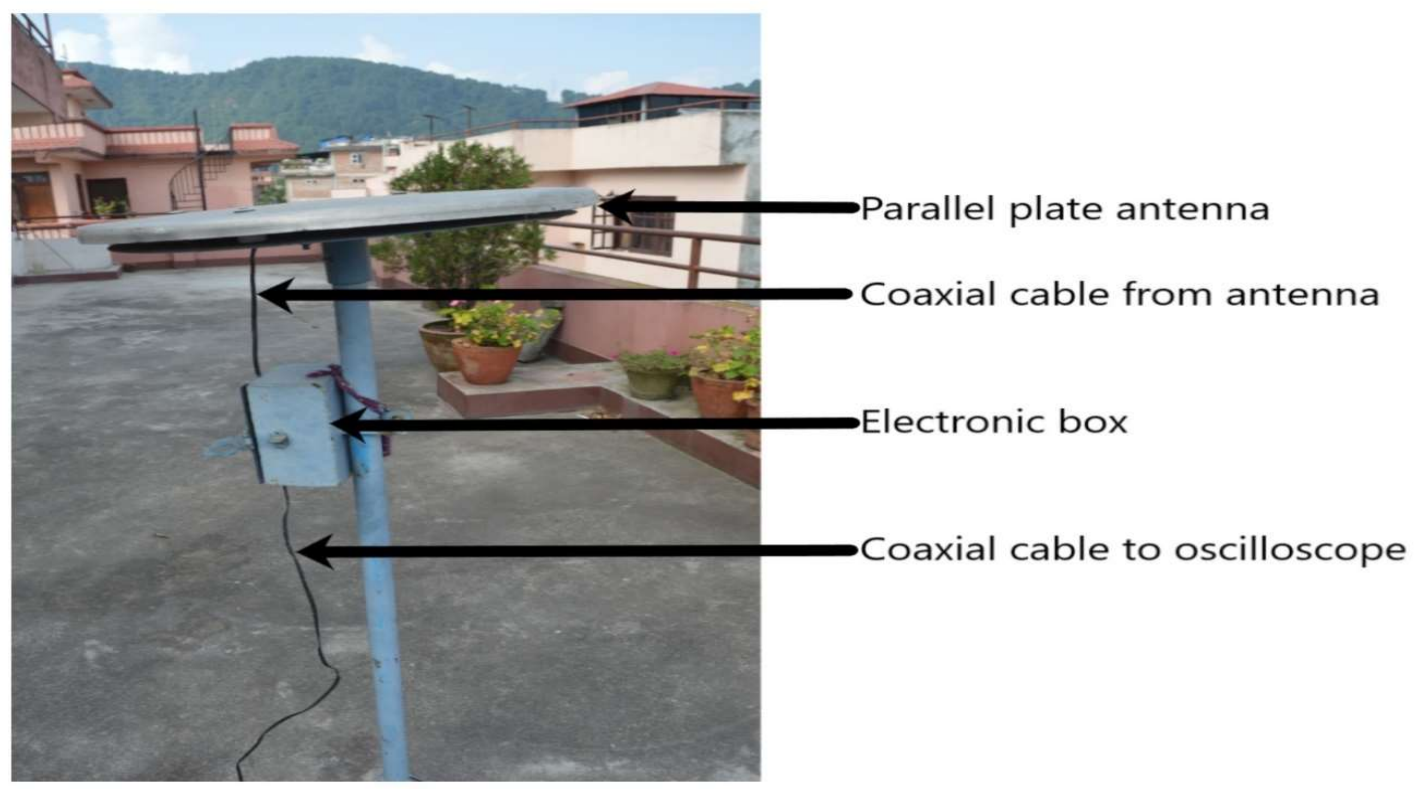

Figure 1: Illustration of the elevated parallel-plate antenna installed in Kathmandu, Nepal.

Wavelet transform algorithms was employed to compute the frequency spectrum of the time domain signal. The signal processed results consist of the time series plot of the electric field signature along with its wavelet transform plot.

\section{RESULTS AND DISCUSSION}

Electric field signatures pertinent to the unusual lightning activity recorded from a measuring station situated in Kathmandu, Nepal have been analyzed using wavelet 
transform algorithms to calculate the frequency contents in those signals. Figure 2 depicts the two examples of the time domain events and their corresponding wavelet transforms use in this research and Table 1 presents the statistical summary of the results of frequency contents. The upper plot on each example of Figure 2 illustrates the time domain electric fields of the unusual event and the lower plot represent its corresponding wavelet transforms. The y-axes (vertical axes) in each upper plot represent the electric field strength in volts (digitizer voltage), whereas the lower plot represents the frequency in the unit of $\mathrm{kHz}$. In both plots, the $\mathrm{x}$-axes (horizontal) represent the time in the unit of second. In each of the plot, time duration of 500 microseconds is taken as the constant window size, where starting point is considered as 0 second. Along with wavelet transform, the data are also subjected to the significance test and cone of influence. The significance test gives the final result's power spectrum above the background noise spectrum, generally white noise and red noise, to a certain confidence level (95\% of confidence level). Moreover, mother wavelet DOG behaves with positive and negative axis values as a separate variable, so wavelet transformed parts or time-frequency plot of the signals are divided into two stages. In this research, the first stage of wavelet transformed signals were correlated with opposite polarity pulse and subsequent part to a main waveform ${ }^{2}$. The frequency range, in which the predominant energy is radiated is termed as spectral range, which is actually the frequency range in which the value of power spectrum is greater than zero. Since, the maximum power of a wave from wavelet transform is not localized at a particular frequency, indeed it is distributed in a range of frequency and is termed as the spread distribution ${ }^{3}$

a)
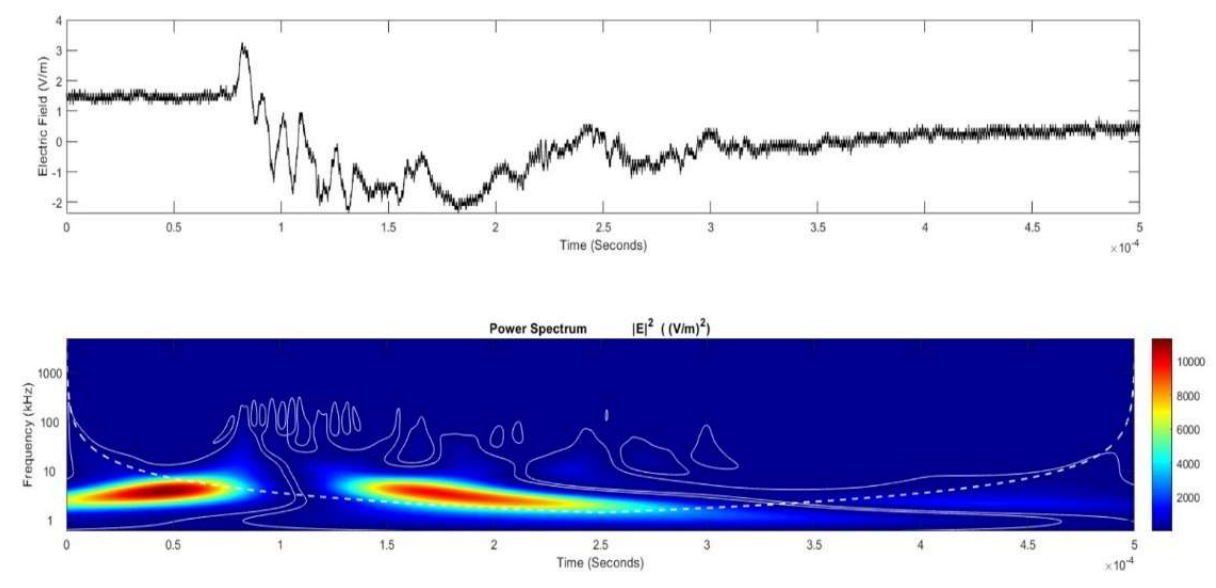

b)
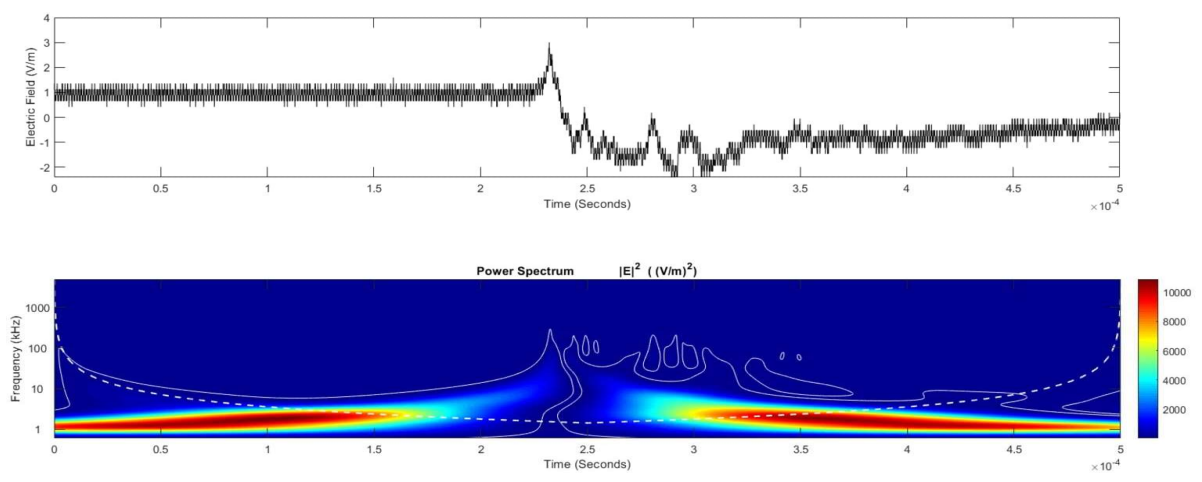

Figure 2: (a) and (b) are examples of electric field waveform analyzed in this paper and its wavelet power spectrum. The upper plot in each figure is the time series of unusual lightning events and the lower is its wavelet power spectrum. 
Table 1: Statistical summary of unusual lightning signals.

\begin{tabular}{|l|l|l|l|l|l|l|l|}
\hline Statistics & $\begin{array}{l}\text { Spectral } \\
\text { (Unusual signals) } \\
\text { range for } \\
\text { opposite } \\
\text { polarity } \\
\text { pulse } \\
\text { distribution } \\
\text { of opposite } \\
\text { polarity } \\
\text { pulse } \\
(\mathrm{kHz})\end{array}$ & $\begin{array}{l}\text { Spread } \\
\text { range of } \\
\text { main } \\
\text { waveform } \\
(\mathrm{kHz})\end{array}$ & $\begin{array}{l}\text { Spread } \\
\text { distribution } \\
\text { of main } \\
\text { waveform } \\
(\mathrm{kHz})\end{array}$ & $\begin{array}{l}\text { Power } \\
\text { peak of } \\
\text { opposite } \\
\text { polarity } \\
\text { pulse } \\
(\mathrm{V} / \mathrm{m})^{2}\end{array}$ & $\begin{array}{l}\text { Power } \\
\text { peak } \\
\text { main } \\
\text { waveform } \\
(\mathrm{V} / \mathrm{m})^{2}\end{array}$ & $\begin{array}{l}\text { Ratio } \\
\text { power peak } \\
\text { polarity } \\
\text { pupposite } \\
\text { main } \\
\text { and } \\
\text { waveform }\end{array}$ \\
\hline Minimum & 1 & 1 & 1 & 1 & 2500 & 1500 & 0.363 \\
\hline Maximum & 400 & 100 & 500 & 95 & 40000 & 50000 & 1.667 \\
\hline Average range & $2-173$ & $4-15$ & $2-162$ & $3-23$ & 15375 & 19218 & 0.984 \\
\hline
\end{tabular}

The statistical figures of Table 1 suggest that the opposite polarity pulse is found to radiate energy predominantly in the average frequency range of $2-173 \mathrm{kHz}$ with a minimum value of $1 \mathrm{kHz}$ to maximum value of $400 \mathrm{kHz}$. The spread distribution of the opposite polarity pulse has a minimum value of $1 \mathrm{kHz}$ to maximum value of $100 \mathrm{kHz}$. The average spread distribution radiated by opposite polarity pulse is in the range of 4-15 $\mathrm{kHz}$. Similarly, the minimum and maximum value of spectral frequency radiated by the main waveform are $1 \mathrm{kHz}$ and $500 \mathrm{kHz}$ respectively. The main waveform radiates energy predominantly in the average frequency range of 2-162 $\mathrm{kHz}$. The spread distribution of the main waveform radiates energy in the average range 3$23 \mathrm{kHz}$. The minimum and maximum value of frequency radiated by spread distribution of main waveform are $1 \mathrm{kHz}$ and $95 \mathrm{kHz}$ respectively. The minimum and maximum value of power peak of the opposite-polarity pulse is found to be $2500(\mathrm{~V} / \mathrm{m})^{2}$ and $40000(\mathrm{~V} / \mathrm{m})^{2}$ with average value of the power peak being $15375(\mathrm{~V} / \mathrm{m})^{2}$. Similarly, the minimum and maximum value of power peak of the main waveform is calculated to be $1500(\mathrm{~V} / \mathrm{m})^{2}$ and 50000 $(\mathrm{V} / \mathrm{m})^{2}$ with the average value of the power peak of the main waveform is $19218(\mathrm{~V} / \mathrm{m})^{2}$. The minimum value of ratio of the power peak of opposite-polarity pulse and that of the main waveform is found to be 0.363 . Furthermore, the maximum value of the power peak of the opposite-polarity pulse and that of the main waveform is 1.667 . The average ratio of the power peak of the opposite polarity pulse and that of the main waveform is found to be 0.984 .

The above results indicate that the main event comprises the multiple events with relatively lower average frequency range, whereas the opposite-polarity pulse is generally a single event radiating relatively higher average frequency range. As is seen from the time series of unusual events, a number of small events embedded in the main event; therefore, one can speculate that there are a number of discharges that contribute to the main events. Whereas, the unidirectional opposite-polarity pulse is mainly due to the discharge of a single event. It can further be speculated that various answering leaders from various ground bound objects contribute to the main events. However, it is not yet clear as to why the main event and the preceding leader have opposite polarity. Nevertheless, the discharge phenomena pertinent to the opposite-polarity leader and various events contributing to the main event are essentially similar.

\section{CONCLUSION}

Wavelet transform technique was employed to extract frequency content of unusual lightning events observed and recorded in Kathmandu, Nepal. These lightning events are 
very new to the lightning community and are not fully understood as of today. This is the first time that the frequency spectrum of such an event has been analyzed. Sixteen electric field signatures pertinent to the unusual lightning events have been analyzed for the frequency content. As is stated earlier, the unusual events comprise two field charges with opposite polarities. The first field charge is apparently similar to the leader of normal negative ground flash. Whereas, the second field charge called the main event has the similar field charge as that of positive ground flash.

From the analysis of frequency spectrum, it is found that the leader types of field change radiate energy in the spectral range of $2 \mathrm{kHz}$ to $173 \mathrm{kHz}$. This range of frequency is not in agreement with that of leaders $(87 \mathrm{kHz}$ to $720 \mathrm{kHz})$ pertinent to the normal ground flashes as reported in the previous work ${ }^{4}$. Meaning that the leader type pulses do not resemble with normal leaders in discharge phenomena. Similarly, the main activity predominantly radiates in the frequency range of $2 \mathrm{kHz}$ to $162 \mathrm{kHz}$, this range of frequency is also not in agreement with that of positive return stroke $(5.5 \mathrm{kHz}$ to $81 \mathrm{kHz})$ as reported in the earlier work $^{4}$. It is therefore, the discharge process associated with main events is different from that of positive return stroke, though electric field signatures appear similar. From this analysis, we can conclude that the unusual activities have different origins than the normal positive or negative ground flashes. Furthermore, these unusual events are unique not only in the perspective of the nature of electric field signature i.e. the main event being preceded by opposite polarity leader type pulses but also in perspective of frequency spectra being radiated by them. Therefore, these discharges must some atmospheric conditions leading to such unique field changes and hence it lays another avenue for the researchers to investigate the origin of such activities. Since, the unusual events were noticed in the temperate region (Uppsala, Sweden) and Sub-tropical zone

(Kathmandu, Nepal), it should have some common source of origin between the two regions.

\section{ACKNOWLEDGEMENTS}

We would like to express our sincere gratitude to the International Science Programme (ISP), Uppsala University, Sweden, for providing the instruments, which is used in this research to record data.

\section{REFERENCES}

1. Li, Q., Li, K. \& Chen, X. 2013. Research on lightning electromagnetic fields associated with first and subsequent return strokes based on Laplace wavelet. Journal of Atmospheric and Solar-Terrestrial Physics. 93:1-10.

2. Adhikari, P.B., Sharma, S., Baral, K. \& Rakov, V. A. 2017. Unusual lightning electric field waveforms observed in Kathmandu, Nepal, and Uppsala, Sweden. Journal of Atmospheric and Solar-Terrestrial Physics. 164:172-184.

3. Mirinda, F.J. 2008. Wavelet analysis of lightning return stroke Journal of Atmospheric and Solar-Terrestrial Physics. 70:14011407.

4. Sharma, S.R., Cooray, V., Fernando, M. \& Miranda F.J. 2011. Temporal features of different lightning events revealed from wavelet transform. Journal of Atmospheric and Solar-Terrestrial Physics. 73:507-515.

5. Esa, M. R. M., Ahmad, M. R., \& Cooray, V. 2014. Wavelet analysis of the first electric field pulse of lightning flashes in Sweden. Atmospheric research. 138:253-267.

6. Torrence, C. \& Compo, G.P. 1998. A Practical Guide to Wavelet Analysis. Bulletin of the American Meteorological Society. 79:61-78

7. Sheshyekani, K., Hazrati, M., Sattari, P., Sadeghi, S. H. H., \& Moini, R. 2006. Real-time detection of lightning electromagnetic field data: a wavelet approach. 17th International Zurich Symposium on Electromagnetic Compatibility, IEEE. pp.413-415.

8. Gou, X., Chen, M., Zhang, Y., Dong, W., \& Qie, X. 2009. Wavelet multiresolution based multifractal analysis of electric fields by lightning return strokes. Atmospheric research. 91:410-415.

9. Nedjah, O., Hussein, A. M., Krishnan, S. \& Sotudeh, R. 2010 Comparative study of adaptive techniques for denoising CN Tower lightning current derivative signals. Digital Signal Processing. 20:607-618.

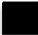

